\title{
ADAPTIVE SMART TV AS A SOCIAL LANGUAGE LEARNING PLATFORM
}

\author{
María José Naranjo Sánchez, Ma Mercedes Rico Garcia, Héctor Sánchez Santamaría \\ and Jesús Salguero Serrat \\ Universtiy of Extreadura, Centro Universitario de Mérida \\ Santa Teresa de Jornet, 38, 06800 Mérida, Spain
}

\begin{abstract}
With the recent emergence of the interactive TV, the iTV starts to gain ground as a learning social media forgotten in the last decades due to previously existing applications (smart phones, tablets, laptops) that surpassed it. The TV has always been used as a means of transmission and family conciliatory, but, at the same time, the necessary feedback, as well as its adaptation to the users' preferences, language level, and age have always been lacking in every learning process that smart televisions can currently provide. In this sense, it seems necessary, on the one hand, to investigate the different characteristics and capabilities of smart televisions and, on the other, to design educational learning pills for English language learning through adaptive smart TVs. To achieve the aforementioned objectives an interactive methodology based on family/ peer gaming will be presented as a way to awake collective and social learning. The expected results will be an application developed with Hybrid Broadcast Broadband technology including a content recommendation system designed considering the user's preferences and a set of educational pills created by a team of linguists and experts in language and methodology. To conclude, this project seeks to promote language learning acquisition in a fun and entertaining way and enhance the experience with their television which has been a traditional model representing bond and time to gather around family and friends.
\end{abstract}

\section{KEYWORDS}

Social Learning, Smart TV, Design, Teaching and Learning, Education

\section{INTRODUCTION}

Today, four screens coexist in many Spanish homes: the television, the computer, the smartphone and the tablet. All these devices have network capabilities, they can access the Internet and communicate with each other, however, for many years TV technology has advanced little beyond its functionality while other devices such as tablets, smartphones, and laptops have risen dramatically. As Catherine Regina et al. (2016) suggest: 'Mobile language learning approaches are clearly in demand and will continue to grow in use as more people turn to smartphones or tablets as a primary computing device.' For this reason, the television has lost its exclusivity as an audiovisual and entertaining device and remained obsolete for the habitual dissemination of multimedia content, although its use continues to be active and frequent in homes, mainly due to its proximity, its place based on family experience, and ultimately, to its social component.

The analogical blackout and the later arrival of the DTT (Digital Terrestrial Television) seemed to draw the favorable scenario for the traditional television channels to develop a new concept of television: interactive TV. However, most audiovisual operators have ignored this functionality of DTT as demonstrated by the almost anecdotal percentage of DTT tuners with MHP (Multimedia Home Platform) that has been commercialized.

These screens, despite the current technological advancement in terms of image and sound quality, allow us to store large amounts of information, interact with the user and operate from a small remote control with a keyboard. Smart televisions nowadays offer us connectivity, making it possible to convert our old television into a complete multimedia entertainment center without missing any other device. 
Thus, the way of enjoying television has changed in the recent years and the possibility of interacting through mobile devices has opened up many paths in the educational field. With regard to the role of foreign languages in education, it is worth mentioning 'The potential of television as an educational tool has been widely recognized in terms of early childhood education, since the launch, for example, of Sesame Street and Play School more than 30 years ago...' (Wood, 2003; Tuula Rajavaara 2005; Pavlov, R \& Paneva, D., 2006; Vásquez, E., \& Bejarano, A., 2011).

Although Television viewing was traditionally the dominant leisure activity for many people (Abadía, I., 2011), there has not been much exposition of language learning resources on TV; in contrast, tablets have become increasingly popular over the past decade due to it multifaceted aspect and variety of tasks that can be undertaken. So, if the television has the appropriate technology to work effectively as a mobile device, we must extend its use to reach the typical motivations for watching TV, such as passing time, companionship, relaxation along with the new added value which is changing the traditional learning style.

Television has been an important device in our daily life for a number of reasons. It is recognized that television is a source of entertainment where family/peers gather, converse, share feelings and thoughts. This social component, seen as its potential, will be the source to enhance the effectiveness of a language learning situation, specially, by presenting real facts that may draw the attention and interest of the users. Furthermore, it can be a powerful learning tool for all age people, particularly teenagers to develop closer attitude and relationships with peers and family members, and at the same time, foster their language learning styles and strengthen their cultural knowledge. Likewise, iTV can also help the elderly who sometimes feel isolated given the fact that the younger generation, the so called digital natives, millennials, or tech savvy are hooked to mobile technology all time and live in a desired social isolation. Thus, these two groups' limitations can be minimized by integrating themselves into the social value of TV intended to entertain the public. This issue is also raised in Renuka Bhattacharjee's work (2017): 'Some television programs are made to raise social awareness. The educational telenovela is one form of edutainment which is very much popular in Latin America. Miguel Sabido has combined communication theory with health and education messages to educate all people throughout Latin America in terms of family planning, literacy, and other current or socially relevant topics.'

According to a project of Northwestern University in the United States (Megan Wood, 2003), it is noted that students are much more likely to retain the knowledge gained from television programs and videos than from lectures. While teachers now make frequent use of other popular technologies such as the Internet and multimedia, it is important to note that television has had a longer and more wide reaching effect on the lives of students. In consonance with S.S Chandra \& Rajendra K. Sharma (2004), one of the most important agencies of informal education is the television; the television has become an important means to informal education in most advanced countries. Most people spend their leisure time in front of the television, and thus absorb a lot of knowledge and information without having to make any great effort. Given these highlights, further development would be more than desirable.

In this light, the STVALL project, funded by the Regional Government of Extremadura (Spain), aims to develop an English teaching platform for interactive televisions which offer personalized training pills to all age users regardless their starting level of English and also taking into consideration their interests and preferences. The platform is based on HbbTV technology because it is available in a great number of televisions in Europe, and particularly, Spain.

This study explores how television as an instructional medium can be integrated with EFL learning and teaching within a social context (at home without setting fixed previous objectives), as well as, its integration in formal education by incorporating newer teaching methodologies based on social and cooperative learning such as Project based-learning, flipped classrooms and gamification under technological environments. In section 1 we have provided an introduction to interactive TV, education, and social learning. The following sections are distributed as follows: section 2 presents the research objectives. Section 3 describes STVALL project including the project's objectives, platform, and learning modes. Section 4 outlines the results, and finally, section 5 summarizes the key findings so far. 


\section{RESEARCH OBJECTIVES}

This study seeks to investigate how the teaching and learning of English can effectively be integrated into Television leisure and education offer. The 2016 NMC Horizon Report on Higher Education in the US (Johnson et al.) lists the blending of formal and non-formal education learning as one of the major challenges in education today. Students are prone to gain easily the necessary skills, knowledge, and motivation to be autonomous learners, being able to use the learning approaches and resources that suit them best. In this light, this device has been always part of everyone's house and we all have sat around to experience a sense of socialization sharing attitudes, thoughts and feelings while watching, discussing and commenting any programme. In addition, the hyper-connected generation, the new potentialities of the mobile devices and the new global vision have given room to fully integrated iTVs. So, our project pretends to:

- $\quad$ 1. Provide new methodologies based on social and collaborative settings.

- 2. Stimulate individual and group learning in an entertaining way.

- 3. Provide flexibility of time and space in learning.

- 4. Reinforce and expand on content being taught.

- 5. Improve the English language

- 6. Increase users' motivation to learn.

- 7. Provide collaborative and social learning to massive audiences

\section{STVALL PROJECT}

As a support of this research, the STVALL project aims, on the one hand, to develop an educational platform for personalized interactive teaching of languages through iTV. The architecture of the platform (see figure 1) is divided into two distinct blocks: (1) the user (client part) that interacts with the intelligent system, and (2) the server where the educational content and individual user profiles are stored, and where the adaptation engine and the author web tool for the construction of training pills are resided. The adaptation engine comprises the recommendation motor and the evaluation system.

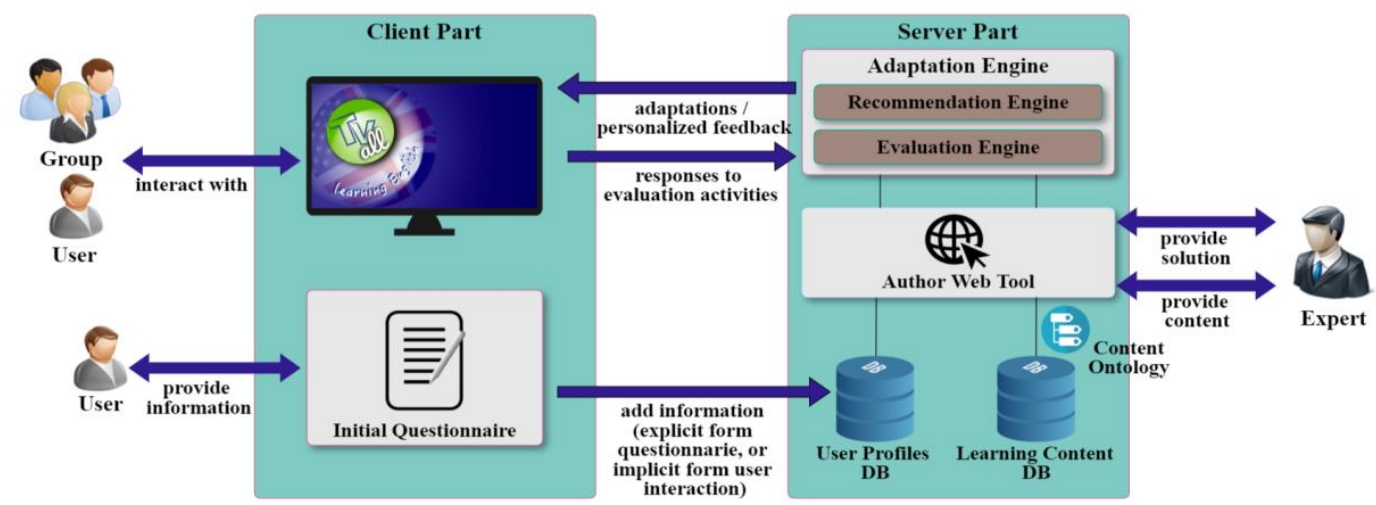

Figure 1. Structure of the STVALL project

\subsection{Platform}

According to the functioning of the platform, the first time users access the platform, they complete an initial questionnaire to create their own profile. The relevant information to create the profile will be formed taking into consideration personal data (full name, age, gender), the English language level they want to reach, along with a selection of topics to determine the user's preferences. Users can choose more than one, these being the options: (1) geography and history; (2) art, literature and culture; (3) science and nature; (4) entertainment, sports, film and television and (5); English language. The information about time devoted 
for the tasks and the ones already done, correct and incorrect answers, attempts of each assignment, score obtained and the percentage of exercises done applied for the skills listening, writing and reading will be recorded. These data allow the recommendation system to work effectively.

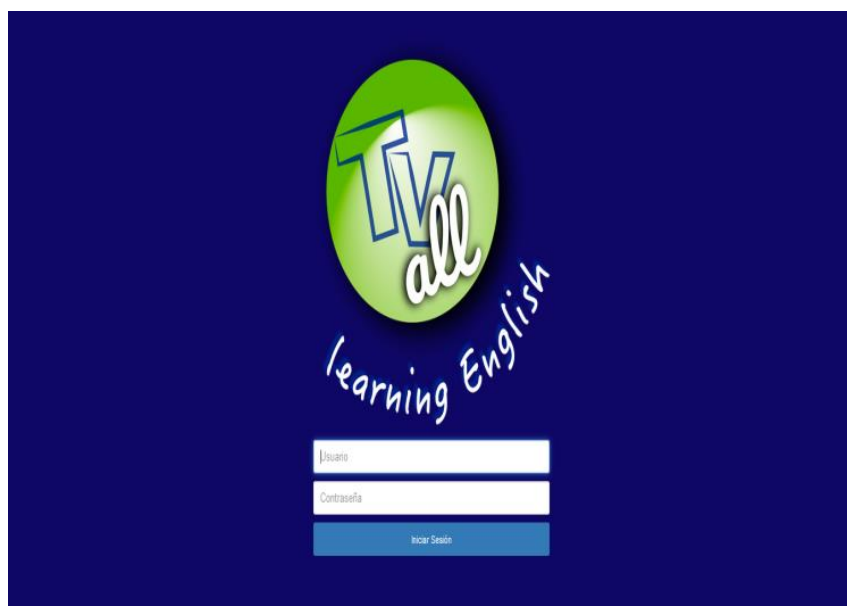

Figure 2. Platform

This system is used to recommend appropriate learning materials to learners based on the learner's profile and also the work carried out by each individual, providing personalization based on their current situation and experience. Additionally, linguists and experts in language and methodology can access the platform to create activities, modify and delete tasks that were previously created. Pending activities in need of feedback will be also corrected and commented by experts.

\subsection{Learning Modes}

The STVALL platform has three learning modes: individual, if it is a single user who accesses the platform (individual access); collaborative if it is a group who access the platform and all of them want to jointly solve the same training activities; and finally, competitive, if the group of students wants to contrast their English language and cultural knowledge (gaming).

As for the individual learning mode, it allows the user to improve their language and cultural level working with the different skills through training activities, particularly, training pills. Once the student accesses this mode, after being completed the questionnaire, the recommendation engine selects a set of assignments that meet the requirements chosen in the previous survey. Each correct task gets a score which is added to the record of the user. In case of mistakes or not completing the task appropriately, the user may improve the score by repeating the activities when the recommendation engine sends the task back again. However, this process occurs when the user ends up the bank of recommended activities for their user model. Undoubtedly, the maximum score assigned for the repeated activity is considerably reduced, getting fewer points than the first time done.

In the collaborative learning mode, again the users fill in the profiles so that the recommendation engine can build a joint user model based on each of the individual involved in the game. As in the previous mode, training pills of different themes and modalities are presented, but in this option they jointly solve them promoting social learning through collaboration and connectivism. When they leave the platform, the engine adds to each individual user model the progress made by the group, adding the points obtained and adding to their history the activities carried out.

Finally, we present the competitiveness mode which is based on group games and which connects with the new active methodologies and where competitiveness, rewards (points or badges) and motivation play an important role in learning. In this case, invited users register and already registered users select their profiles to start the activities regarding their user model. The learning of the lower level student will be favoured when different profiles are created; and the most common topics to all the members of the group will be more likely to be displayed, but without discarding the tasks with preferred themes for each of the individual 
members. Each participant plays in their turn adding points provided correct answers. The user who first reaches the maximum score will be the winner of the game. Once finished, the engine stores the progress of each registered user, adding the training pills made to their history and adding the points won to their total score.

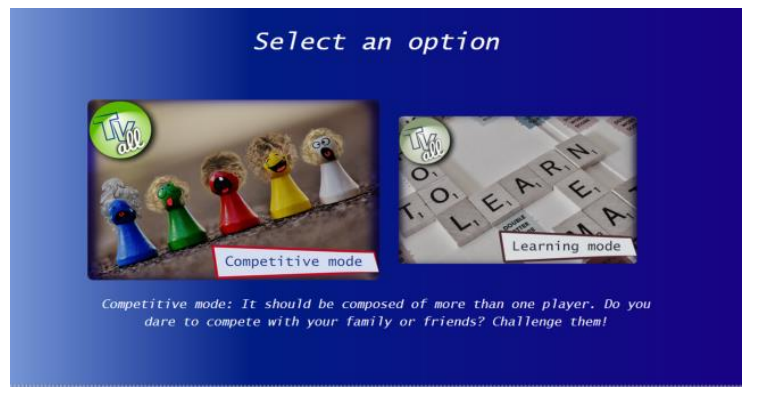

Figure 3. Learning modes

\section{RESULTS: LANGUAGE LEARNING DESIGN THROUGH ADAPTIVE ITV}

To complete the whole project, different research techniques were used to obtain the data for this study. These included a previous study of the literature of adaptive and interactive TV carried out by the department of Computer Systems and Telematics Engineering, followed by the development of the English teaching platform based on HbbTV technology. The language learning platform is the main result for the objectives 1, 2, 3, 6, 7 mentioned in section 2 .

The remaining objectives 4 and 5 lead to creating content to be taught to improve the English language and likewise expand the knowledge providing collaborating and social learning to massive audiences. Thus, for the linguistic and culture content, the language experts selected the topics for the training pills, designed the activities and assessed them. The selected areas of study are:

1. Geography and history;

2. $\quad$ Art, literature and culture;

3. Science and nature;

4. Entertainment, sports, film and television and;

5. English language.

Our purpose as language specialist is to work with learning pills to foster the communicative and social approach that can be maximized from the iTV since learning a language is about more than just memorizing vocabulary lists and verb tense forms.

From the methodological approach, we adhere to the ecological theory (Van Lier, L., 2012) of language learning, dynamic games (in any of the modalities), collaborative learning and connectivism, adaptation of the contents to the user profiles according to their age, preferences and English level.

Hence, the team of language experts has been responsible for feeding the platform with around 500 training activities in each mode (interactive and non-interactive activities) with a total of around 1000 tasks. For each activity the system stores the subject (the 5 categories mentioned above), the linguistic level required by the user according to the Common European Framework of Reference for Languages (A1/A2, B1/B2), the modality (reading, listening, speaking, writing), the type of discourse (monological or dialogical), the type of text (narration, description, argumentation, instructions, biographies, advertising, informative or comic texts), and if it is oriented to child or adult audience.

As noted before, another function of the team of experts is to assess the evaluation activities with pre-recorded feedback. The expert will receive notifications of pending evaluation activities when accessing the platform. Immediate solutions will be provided to users, allowing, in addition, a more personalized and motivating learning. The training activities for each of the topics mentioned above are classified in:

- Non-interactive or presentation mode activities, where the student only visualize the training content offered in the activity (listening and reading). These activities are short (the videos last around one minute, and texts are brief, no more than 150 words) to avoid unnecessary scrolls in the screen which will hinder the purpose of the training pills and iTV functionality. No feedback is included. 


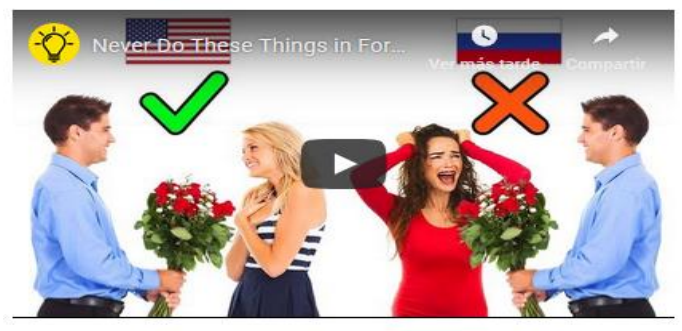

Figure 4. Non-interactive activity

- Interactive or evaluation with immediate feedback tasks, where the student must provide some answer to the question arisen. The feedback can be offered by the evaluation engine without the intervention of the expert. When presenting speaking and writing activities, the feedback can be given by peers, promoting self-evaluation or by experts.

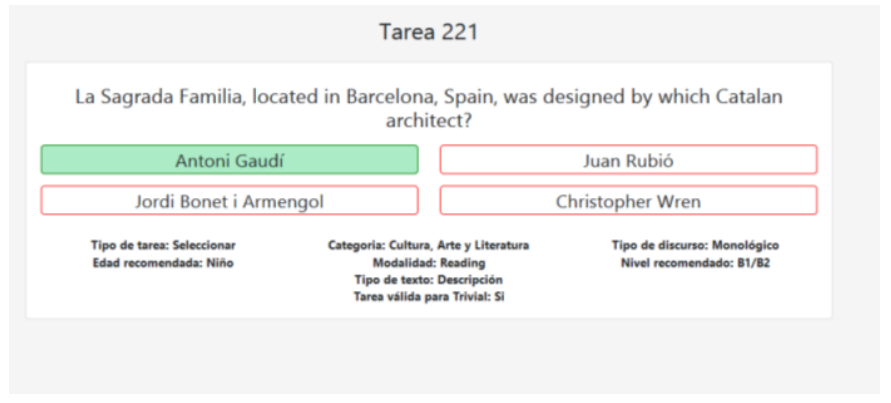

Figure 5. Interactive activity with immediate feedback

- Interactive or evaluation with pre-recorded feedback activities, where feedback cannot be automatically offered by the evaluation engine without the intervention of the human expert (long texts and speaking).

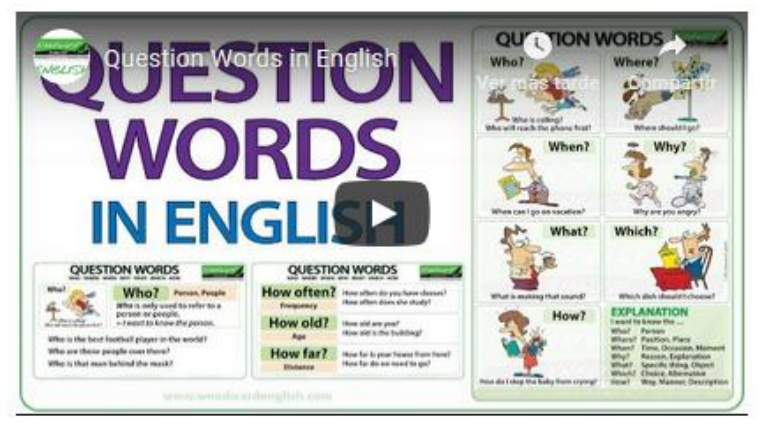

Figure 6. Interactive activity with pre-recorded feedback

The language experts have designed the following types of activities considering that pills are short inputs aiming at entertaining the users: activities with resources: image, video, audio (around 30 and 50 seconds); matching exercise; fill in the blank spaces; select the correct answer for the interactive tasks with immediate feedback. For non-interactive activities the ones presented are video (no more than 60 seconds) and text input. 


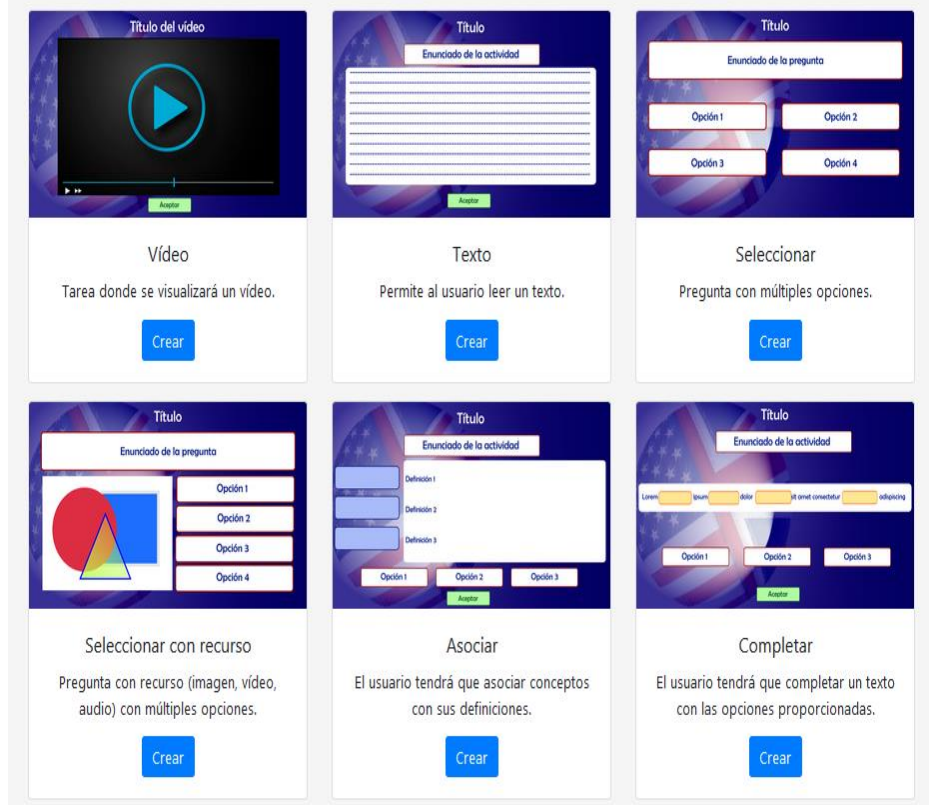

Figure 7. Different types of activities designed

After the review of literature about the strengths and weaknesses of mobile applications in comparison to the iTV, focusing on their trends, challenges and opportunities and our depiction of how the teaching and learning of English can effectively work into Television leisure and education, we hope to offer qualitative and quantitative results about the learning improvement of the sample that will be tested in short. Similar research justifies the learning improvement of t-learning applications developed (Dos Santos, D.T. et al, 2006; Souza, Maicon \& Bizelli, José, 2012).

\section{CONCLUSION}

Although television has always been seen as a tool of indirect teaching and learning in the past- video presentations, films, series, and any kind of input have been used to promote intellectual and cultural growth in classrooms-, the originality of the proposal lies in the analysis of the effectiveness and use of educational content through television, in an environment that allows interactive, group and personalized learning within the scope of any user. In the last few years, teaching through television has become more popular and has reached a higher level of sophistication. For that purpose an English teaching platform based on HbbTV technology for interactive television offering personalized training pills to a user or a group of users have been created. The findings may reveal that users acknowledge the importance of using TV as a means of transmission and family/peer conciliatory. In addition, it meets the requirements of the traditional use of TVs, the socializing factor. Currently, we cannot provide quantitative results since this proposal is in its first phases; qualitative and quantitative results will be studied soon. Thus, further lines of research may lie in the implementation of the iTV with a social purpose in public or private channels and a later the analysis of user's data and so that all citizens have the opportunity to acquire skills and attitudes, promoting the sustainable development of knowledge and values through educational content in an interactive environment.

\section{ACKNOWLEDGEMENT}

This project has been co-financed by the Ministry of Economy and Infrastructure of Junta de Extremadura and by the European Regional Development Fund (ERDF) of Extremadura (Ref. IB16160). 


\section{REFERENCES}

Abadía, I. 2011. Revisión de lineamientos para el desarrollo de contenido educativo para televisión digital interactiva. Revista S\&T, 10(20), 71-104.

Dos Santos, D.T. et al, 2006. Digital TV and Distance Learning: Potentials and Limitations. Proceedings - Frontiers in Education Conference. Conference: Frontiers in Education Conference, 36th Annual. DOI: 10.1109/FIE.2006.322670

Johnson, L. et al. 2016. NMC Horizon Report: 2016 Higher Education Edition. Austin, Texas: The New Media Consortium. Retrieved from http:/www.nmc.org/publication/nmc-horizon-report-2016-higher-education-edition/

Pavlov, R. \& Paneva, D., 2006. Interactive TV-based learning, models and standards. HUBUSKA Open Workshop Semantic Web and Knowledge Technologies, 70-99. Varna.

Rajavaara, Tuula, 2005. Television ... as a tool of learning. Examples from the Finnish broadcaster, YLE.

Regina C. et al, 2016. A review of mobile language learning applications: trends, challenges and opportunities. Eurocall Review. Retrieved from https://polipapers.upv.es/index.php/eurocall/article/view/6402/7213

Renuka, B., 2017. International Journal of Innovative Research and Advanced Studies (Ijiras). 178-183. Retrieved from http://www.ijiras.com/2017/Vol_4-Issue_1/paper_37.pdf

S.S Chandra \& Rajendra K. Sharma, 2004. Sociology of Education. Delhi: Atlantic Publishers and Distributors.

Sabido, M., 1989. Soap operas in Mexico. Paper presented to the Entertainment for Social Change Conference, at Annenberg School of Communications, University of Southern California, Los Angeles.

Souza, Maicon \& Bizelli, José, 2012. Televisão digital interativa na educação: desenvolvimento de um conteúdo complementar ao ensino. Revista Ibero-Americana de Estudos em Educação, ISSN-e 1982-5587, Vol. 7, No. 1, 2012, págs. 84-97.

Van Lier, L., 2012. Language learning: An ecological - semiotic approach. In E. Hinkel (Ed.), Handbook of second language teaching and learning (Second Edition). Mahwah, NJ: Erlbaum.

Vásquez, E., García, A., \& Bejarano, A., 2011. Contenidos Educativos Para Televisión Digital. Sesiones LACLO2011 - SESION 13: Desarrollos de Contenidos.

Wood, M., 2003. Using television to educate, stimulate and disseminate. Curriculum and Leadership Journal. 